REGARDS

SUR LECONOMIE ALLEMANDE

BULLETIN ECONOMIQUE DU CRAC

\section{Regards sur l'économie allemande}

Bulletin économique du CIRAC

$81 \mid 2007$

Varia

\title{
Une croissance solidement assise
}

Isabelle Bourgeois

\section{(2) OpenEdition}

Journals

Édition électronique

URL : http://journals.openedition.org/rea/647

DOI : $10.4000 /$ rea. 647

ISBN : 978-2-8218-0858-4

ISSN : 1965-0787

Éditeur

CIRAC

Édition imprimée

Date de publication : 1 mai 2007

Pagination : 3-4

ISSN : 1156-8992

Référence électronique

Isabelle Bourgeois, « Une croissance solidement assise », Regards sur l'économie allemande [En ligne], 81 | mai 2007, mis en ligne le 17 juin 2008, consulté le 15 septembre 2020. URL : http:// journals.openedition.org/rea/647 


\section{Une croissance solidement assise}

L'Allemagne semble avoir renoué durablement avec la croissance. Après une année 2006 où, contre toute attente, le PIB a frisé une hausse de $+3 \%$, les Instituts avaient commencé à revoir à la hausse leurs prévisions pour 2007. La bonne tenue confirmée de la conjoncture depuis le début de l'année les amène à redoubler d'optimisme : selon les estimations de leur rapport de printemps, le PIB devrait croître de +2,4\% en 2007 comme en 2008 - et ce, malgré la hausse de la TVA. Même le gouvernement fédéral, qui tablait jusqu'ici sur $+1,7 \%$ pour 2007 , vient de porter ses prévisions à $+2,3 \%$ (et $+2,4 \%$ en 2008).

L'économie allemande, championne du monde à l'export, bénéficie toujours d'un marché mondial dynamique et porteur. Mais bien plus encore, elle tire désormais les fruits d'une politique de longue haleine et, surtout, collective, dont les effets se sont cumulés au fil du temps et lui ont permis de consolider sa compétitivité. Les partenaires sociaux ont veillé à préserver la productivité des entreprises grâce à une modération salariale unique en Europe sur la décennie écoulée. Les entreprises ont mis à profit la crise consécutive à l'éclatement de la bulle Internet de 2000 pour accroître leur rentabilité en menant des restructurations drastiques. Elles avaient certes nourri la hausse du chômage, mais leur profitabilité retrouvée se traduit aujourd'hui par une nette reprise de l'embauche. Le gouvernement fédéral, pour sa part, a entrepris d'agir sur le cadre des activités, mettant en œuvre progressivement depuis 2003 la Stratégie de Lisbonne. Cette politique favorable à l'activité a enclenché une décrue du chômage bénéfique aux budgets sociaux comme au volume des revenus. La reprise de l'emploi contribue ainsi à tirer une demande intérieure qui conforte sa fonction de second moteur conjoncturel. La dynamique de la croissance et de l'emploi se traduit par une nette augmentation des recettes fiscales qui permet aujourd'hui à l'Allemagne de respecter les critères du Pacte de stabilité et de croissance et de présenter à brève échéance un budget équilibré.

C'est donc la demande intérieure qui tire désormais la croissance. Alors qu'elle a contribué pour plus de la moitié à la hausse du PIB en 2006 (1,5 point de pourcentage contre 1,1 point seulement pour les échanges extérieurs), sa part devrait s'accroître encore en 2007 et 2008 et atteindre respectivement 1,7 et 2 points de pourcentage. En 2006 déjà, la consommation des ménages était en hausse : $+2,1 \%$ selon Destatis. Pour la première fois depuis 2000, leurs dépenses ont crû plus vite que leur revenu disponible $(+0,8 \%)$, ce qui s'est accompagné d'une baisse du taux d'épargne (passé de 10,6\% à 10,5\% en 2006). Or les ménages, après avoir effectué leurs achats de biens de consommation durable avant la hausse de la TVA, passée de 16 à $19 \%$ au 1er janvier 2007, ont consacré les trois premiers mois de l'année à reconstituer leur épargne. Selon les Instituts, l'effet TVA aura ainsi tout au plus légèrement retardé durant le premier trimestre 2007 la montée en puissance de la consommation. Car celle-ci repose sur l'augmentation du revenu réel, conséquence directe d'une reprise des activités qui, maintenant, tire effectivement l'emploi.

Depuis 2006, la situation sur le marché de l'emploi ne cesse en effet de s'améliorer, portée par une progression des coûts salariaux horaires $(+0,8 \%)$ faible en comparaison de celle de la productivité $(+2,0 \%)$. Le nombre d'actifs occupés s'est accru de 300000 en moyenne sur l'année écoulée ; mieux encore, les emplois soumis à cotisations sociales sont majoritaires, les 'petits boulots' étant en net recul. D'ici 2008, le nombre de chômeurs devrait redescendre à 3,5 millions, une estimation partagée par les Instituts et le gouvernement fédéral. Traduisant la flexibilisation en cours du marché, l'emploi temporaire se révèle particulièrement dynamique (un tiers des embauches). Or si les statistiques officielles le comptabilisent dans le tertiaire (les entreprises d'intérim entrent dans la catégorie des services aux entreprises), c'est en réalité l'industrie qui embauche les intérimaires. Celle-ci, qui affronte un niveau de commandes comparable à celui des années du boom de l'unité allemande et dont le taux d'utilisation des capacités atteint le record de $87,5 \%$ selon l'Institut IfW de Kiel, manque en effet de main d'œuvre.

L'industrie, qui occupe 7,4 millions d'emplois (19\% du total allemand), a contribué en 2006 pour un quart à la création de richesse (24\%, soit 490 milliards $€$; Destatis) ; la construction mécanique y contribuant à elle seule pour $15 \%$. Alors que, depuis 1970 , la productivité de l'économie allemande enregistrait une hausse de $+1,7 \%$ en moyenne annuelle, elle était de $+2,7 \%$ dans l'industrie ; à l'inverse, les coûts salariaux unitaires y ont crû moins vite $(+2,3 \%$ en progression annuelle contre $+2,5 \%$ en moyenne allemande). Depuis quelques années, ils tendent même à baisser. Compétitive dans la concurrence mondiale, l'industrie allemande continue d'étendre ses capacités pour répondre à la demande, comme en atteste la hausse continue des investissements en biens d'équipement.
Les fruits d'une politique collective

Rôle primordial de la demande intérieure

Reprise de l'emploi

Une industrie prospère et qui investit 


\begin{tabular}{|c|c|c|c|c|}
\hline \multicolumn{5}{|c|}{ Prévisions du rapport de printemps 2007 des Instituts de conjoncture } \\
\hline & 2005 & 2006 & 2007 & 2008 \\
\hline \multicolumn{5}{|c|}{ (variation en \% par rapport à la même période de l'année précédente) } \\
\hline $\begin{array}{l}\text { PIB }{ }^{1)} \\
\text { Ouest (Berlin inclus) }{ }^{2)} \\
\text { Est }{ }^{2)} \\
\text { Consommation privée } \\
\text { Consommation publique } \\
\text { Investisssements bruts en biens d'équipement } \\
\text { biens d'équipement. } \\
\text { construction } \\
\text { autres } \\
\text { Demande intérieure } \\
\text { Exportations } \\
\text { Importations } \\
\text { Prix à la consommation (2000 = indice 100) } \\
\text { Coûts salariaux unitaires }\end{array}$ & $\begin{array}{r}0,9 \\
1,0 \\
-0,2 \\
0,1 \\
0,6 \\
0,8 \\
6,1 \\
-3,6 \\
4,7 \\
0,5 \\
6,9 \\
6,5 \\
2,0 \\
-1,1\end{array}$ & $\begin{array}{r}2,7 \\
2,6 \\
3,0 \\
0,8 \\
1,8 \\
5,6 \\
7,3 \\
4,2 \\
5,9 \\
1,6 \\
12,5 \\
11,1 \\
1,7 \\
-1,3\end{array}$ & $\begin{array}{r}2,4 \\
2,4 \\
2,4 \\
0,9 \\
1,1 \\
4,3 \\
6,2 \\
2,7 \\
5,9 \\
1,8 \\
8,4 \\
7,5 \\
1,8 \\
-0,1\end{array}$ & $\begin{array}{l}2,4 \\
2,4 \\
2,4 \\
2,1 \\
1,3 \\
4,3 \\
6,2 \\
2,7 \\
4,9 \\
2,1 \\
8,1 \\
8,4 \\
1,8 \\
1,5\end{array}$ \\
\hline \multicolumn{5}{|c|}{ (chiffres nominaux) } \\
\hline $\begin{array}{l}\text { Déficit public en \% du PIB } \\
\text { Dette publique (en milliards } € \text { ) } \\
\text { Actifs (en millions) } \\
\text { Chômeurs indemnisés (en millions) }{ }^{3)}\end{array}$ & $\begin{array}{r}-3,2 \\
-72,6 \\
38,8 \\
4,9\end{array}$ & $\begin{array}{r}-1,7 \\
-39,5 \\
39,1 \\
4,5\end{array}$ & $\begin{array}{r}-0,6 \\
-13,3 \\
39,6 \\
3,8\end{array}$ & $\begin{array}{r}0,0 \\
0,0 \\
39,8 \\
3,5\end{array}$ \\
\hline
\end{tabular}

Source des données : Rapport de printemps des six Instituts de conjoncture, en date du 19-04-2007 (les prévisions 2007 et 2008 prennent pour hypothèse notamment un prix moyen du baril de Brent de $65 \$$, un taux de 1,32 $\$=1 €$, et une croissance du commerce mondial de $7,5 \%$ en 2007 comme en 2008). 1) aux prix de l'année précédente. 2) état : février 2007. 3) chiffres arrondis par nos soins. NB : ces données ne sont que partiellement comparables à celles du rapport d'automne (voir REA 79/06) étant donné la révision/actualisation constante des calculs effectuée par les Instituts.

Dynamisation des activités à l'est aussi
L'embellie atteint également les nouveaux Länder, où la croissance industrielle a été supérieure à celle de l'ouest. Alors que les salaires y sont inférieurs de $33 \%$ depuis de nombreuses années, l'écart de productivité s'est réduit à $22 \%$, expliquent les Instituts. Le regain touche même le BTP qui, à l'ouest comme à l'est, a vu sa situation se normaliser ; les investissements reprennent. Plus important, les surcapacités accumulées dans les années 1990 profitent aujourd'hui à l'économie, permettant aux entreprises de s'étendre. A l'est aussi, la reprise décrispe le marché de l'emploi : pour la première fois depuis onze ans, l'emploi soumis à cotisations sociales est en hausse, surtout dans l'industrie et les services aux entreprises. Mais les Länder est-allemands n'ont pas encore achevé leur rattrapage.

L'AllemAGNE EST REDEVENUE LA 'LocomotiVE' DE L'EUROPE. Mais si sa croissance est solide, elle n'est pas encore durable. II existe en effet un certain nombre de risques, moins externes qu'internes. Le premier consiste à ralentir les réformes visant à débrider la dynamique économique. Second risque : si la hausse des recettes fiscales induite par la croissance a permis d'abaisser considérablement le déficit, le budget allemand est loin d'être structurellement consolidé. Les Instituts se montrent plus optimistes que le gouvernement fédéral sur la réduction du déficit, ce dernier ne fixant le retour à l'équilibre budgétaire qu'à I'horizon 2010. Divers ministères souhaitent en effet lancer des programmes dont le coût total s'élèverait à quelque 7 milliards $€$; or pour Michael Glos comme pour son collègue des Finances, la réduction des dépenses de consommation du Bund doit avoir priorité absolue. L'Allemagne doit aussi assumer son rôle de modèle au sein de l'Eurogroupe, a rappelé Peer Steinbrück, afin de consolider la confiance dans l'euro. Au plan intérieur, la réduction du déficit structurel s'impose d'urgence pour contrefinancer la hausse des investissements dans la formation, l'innovation et la modernisation des infrastructures, de même que la poursuite de la baisse des prélèvements sociaux (la hausse de la TVA qui a abaissé de $6,5 \%$ à 4,2 \% du salaire brut les cotisations chômage n'est qu'un premier pas), voire fiscaux. Mais la réforme de la fiscalité que préconisent les Instituts dans leur rapport est prématurée tant que le budget allemand ne sera pas assaini, rappelle le gouvernement fédéral. Troisième risque enfin : la tentation grandissante de redistribuer les fruits de la croissance, comme le révèlent l'actuel débat sur l'introduction d'un salaire minimum légal ou la revendication d'une hausse salariale de 6,5\% dans les négociations en cours dans la métallurgie. Si la première mesure se révélerait «dommageable à l'emploi » (Instituts), la seconde romprait avec la modération salariale à laquelle l'économie allemande doit en grande partie sa reprise, comme le rappelait avec insistance la Bundesbank dans son rapport de mars. Surtout, expliquent les Instituts, elle se répercuterait invariablement sur l'inflation, forçant la BCE à accroître ses taux à un niveau qui alors pourrait se révéler restrictif pour l'économie allemande. 